\title{
Regenerative medicine techniques in cardiovascular disease: where is the horizon?
}

Regenerative medicine techniques to restore cardiac and vascular function are being increasingly investigated as management options for cardiovascular disease. The authors set out to identify emerging regenerative techniques in cardiovascular disease and investigate their stage of development. The relevant networks in the field in the UK were contacted and online sources for cell therapy, tissue engineering, and other regenerative techniques and products were searched for online. A total of 49 Phase II, II/III and III trials of regenerative products or techniques were identified: 13 Phase III, eight Phase II/III and 28 Phase II trials. Twelve of the Phase III trials are for myocardial ischemia and involve an intracoronary infusion or intramyocardial injection of autologous bone marrow-derived stem cells. Most of those in Phase III trials are, however, associated either with an unproven delivery technique or cellular approach. The authors conclude that translation into clinical practice and diffusion into health systems is some way off.

\section{KEYWORDS: cardiovascular disease factor-based therapy regenerative medicine stem cell therapy}

Despite recent reductions in incidence and improvements in preventive and acute management, cardiovascular disease (CVD) remains responsible for significant health and social costs, and productivity losses. CVD, and in particular coronary artery disease, is also among the leading causes of death in many countries. Regenerative medicine techniques to restore cardiac and vascular function are being increasingly investigated as viable management options for CVD. The potential capacity of adult and embryonic stem cells to develop into cardiac stem cells, cardiomyocytes, vascular endothelial cells and smooth muscle cells; and to secrete cytokines and growth factors to support endogenous repair, is under evaluation $[1,2]$. In addition, developments in factor-based therapy to induce the body's own regeneration processes via the action of paracrine factors, are also gaining attention as potential therapeutic options [1].

Support for progressing developments in regenerative medicine is gathering pace in a number of countries throughout Europe and the developed world [3-10]. The commitment to fund both basic science and translational research should mean that early research in regenerative medicine in CVD, which once appeared a distant vision, can be transformed into reality. In 2012, Ptaszek et al. deemed development of regenerative therapeutic strategies to reverse the progression of advanced heart failure one of the most urgent clinical needs of the 21st century, and concluded that an ideal cardiac regenerative therapy would involve a key cell type, a paracine factor, a cardiac tissue niche and a safe, minimally invasive administration procedure to introduce the therapy to the affected area [11].

The choice of cell source is a fundamental question in cellular therapy. Using autologous cells may well be safe from adverse immune response, but has limitations, including the decline of regenerative capacity with age, the need for a collection procedure in people with serious underlying conditions and patient-topatient variability in response [12]. In addition, although relatively costly, there are no intellectual property rights in the cell collection, isolation and reinfusion techniques, as they are generally standard procedures. Allogenic cells from younger, healthy donors have a higher regenerative potential than older autologous cells and cells from one donor can be used for more than one patient. Therefore, allogenic cells have a greater commercial value and the possibility of developing an 'off-the-shelf' product. However, although allogenic mesenchymal stromal cells, a subgroup of the bone-marrow derived mononuclear cells, are thought to be predominantly immunosuppressive, there are reports that such cells can switch from an immunomodulatory state (no generation of an immune response in the recipient) to an immunostimulant state with the potential to generate an immune response [13]. If this observation is confirmed then an immunosuppressant regimen may be required postimplantation, increasing the risks of adverse reactions.

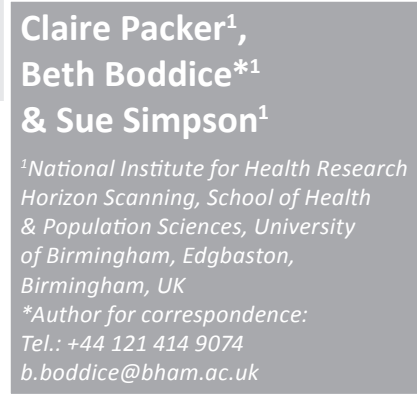

\section{(6) Regenerative Medicine}


The complete mechanism of action of stem and progenitor cell regeneration is uncertain, but does not appear to be solely operating through the replacement or regeneration of lost cells. There is evidence that infused stem cells induce paracrine cell-to-cell signaling, such as the production of cytokines or other factors, which may increase endogenous cellular repair and support increased collateral blood vessels [13]. An inverse relationship between benefit and cell dose administered has been reported and needs further elucidation [14]. An additional complicating factor for evaluation is the implantation method, which may have different efficacies for different patient groups. Current trials use either an intracoronary infusion or direct intramyocardial injection.

To date, there has been conflicting evidence on the ability of regenerative medicine techniques to improve ventricular function. In 2012, a Cochrane review updated evidence from 33 randomized, controlled trials (1765 participants) evaluating the effectiveness of autologous stem or progenitor cells to improve cardiac function in patients with acute myocardial infarction [15]. The authors found that cell therapy was not associated with statistically significant changes in the incidence of mortality (relative risk: 0.70; 95\% CI: $0.40-1.21$ ) or morbidity as measured by reinfarction, hospital readmission, restenosis and target vessel revascularization. However, despite the significant heterogeneity of the studies included, data demonstrated a short-term improvement in left ventricular ejection fraction (LVEF; weighted mean difference: $2.87 \%$; 95\% CI: 2.00-3.73) that was maintained over 12-61 months. The reviewers concluded that larger trials are required as well as standardization of methodology, cell dosing, formulation, timing of cell transplantation and patient selection.

In the work reported here, the authors identify and characterize emerging applications of regenerative medicine (stem cells and factor-based therapies) in development for the management of CVD and examine their stage of development for specific patient indications. This work was undertaken to inform health service policy- and decision-makers in England of key developments, in order that the development of national guidance can be undertaken in a timely fashion.

\section{Methods}

The authors undertook the initial searching for their review between June 2011 and January 2012, identifying key networks, organizations, experts and companies currently active in the regenerative medicine field. A list of commercial products known to be in development for CVD from the univerCellmarket database [101] was supplemented with a search of online sources (Box 1) to identify other regenerative techniques and products. Searches were updated in February 2013.

Medline was searched from 1996 to week 2 January 2012 (updated for clinical trials to week 5 February 2013). ClinicalTrials.gov searching was limited to trials first received between January 2001 and January 2012, with the status of Phase II and III trials updated in February 2013. The searches used a mix of medical subject headings and keywords including cardiac, heart, regenerative medicine, cardiovascular stem cell, cell therapy, tissue engineering and regeneration. The authors initially searched for any cell therapy, tissue engineering, and regenerative techniques and products being developed for any CVD indication in any clinical trial phase. We excluded any approaches or products that were in, or had reported results from, proof-of-concept or Phase I trials, but had no indication of ongoing Phase II trials.

For each identified approach or product thought to be in Phase II or III trials, or closer to licensing, the relevant commercial or academic institutes developing the technology were identified. Information was then collated about their approach, delivery method, specific clinical indication, ongoing trials and results from completed trials. The techniques found to be in Phase II, II/III and III trials are reported on here.

\section{Results}

The authors identified 49 Phase II, II/III or III clinical trials; a third in association with commercial developers (Figure 1). The majority of Phase II, II/III and III trials associated with industry are in development in the USA.

Three noncellular, factor-based therapies were identified: one in Phase III (adenovirus serotype- 5 vector to deliver human fibroblast growth factor 4; trial 2; Table 1) and two in Phase II trials (FGF-1 and thymosin $\beta$-4; trials 17 and 21; Supplementary Table 1; see online www.futuremedicine.com/doi/suppl/10.2217/RME.13.21). The authors found two products using allogenic cells in Phase II trials (Revascor ${ }^{\circledR}$ and Prochy$\mathrm{mal}^{\circledR}$; trials 19 and 20; Supplementary Table 1). All the remaining trials reported using autologous cells that, except for three, were all derived from bone-marrow aspiration. Seven of the 12 cellbased Phase III trials used mononuclear cells, one used mesenchymal cells, one isolated and used CD $34^{+}$cells, two isolated and used CD133+ 
Regenerative medicine techniques in cardiovascular disease: where is the horizon? REVIEW

Box 1. Online sources searched to identify developments.

- Bibliographic databases: Medline, Embase and the Cochrane research database

- Regulatory authorities: EMA and US FDA

- Research funders: UK research councils and EU Framework Programme for Research consortia

- Global trial registry of ongoing research: ClinicalTrials.gov

- UK regenerative medicine organizations and networks

cells in combination with coronary artery bypass grafting $(\mathrm{CABG})$ and one transformed mesenchymal cells into cardiopoietic stem cells (C-Cure ${ }^{\circledR}$ from Cardio3 BioSciences, Belgium; trial 9; Table 1).

\section{Clinical indications \& delivery}

Of the 13 Phase III trials, three are trials for refractory chronic angina; five for use in patients' postacute myocardial infarction (three in combination with $\mathrm{CABG}$ ); three for ischemia-related heart failure; and two for dilated cardiomyopathy from other causes. Of the Phase III trials, eight involve, or will involve, an intramyocardial or transmyocardial injection, and five an intracoronary infusion.

Of the 36 Phase II/III and II trials (SUPpiementary Tables $1 \& 2$ ), one is for ischemic stroke, four for nonischemic dilated cardiomyopathy, three for refractory angina, 16 for use following acute myocardial infarction, and the rest for ischemic cardiomyopathy and heart failure. Delivery is by intramyocardial injection or intracoronary infusion for all trials except one allogenic product and one factor-based product, which are delivered intravenously (Prochymal, thymosin $\beta-4$; trials 20 and 21; Supplementary Tabie 1), and the trial in ischemic stroke (trial 18; Supplementary Table 1), which uses an intracarotid administration.

\section{- Phase III trials \\ Refractory angina}

CD $34^{+}$autologous bone marrow-derived stem cell therapy (Baxter Healthcare Corporation, IL, USA), in development for the treatment of refractory angina, recently entered a Phase III trial. $\mathrm{CD} 34^{+}$cells are a subset of mesenchymal stem cells and are thought to be involved in the creation of new blood vessels and increased tissue perfusion. The RENEW Phase III trial [102] aims to enroll 450 patients across the USA and will compare intramyocardial delivery of $\mathrm{CD} 34^{+}$autologous cells harvested after G-CSF mobilization and apheresis with standard care or placebo. The trial is expected to last up to 24 months and has a primary end point of exercise tolerance at 12 months. Secondary end points include frequency of angina episodes and major cardiac events. In a Phase II trial involving 167 patients with refractory angina, those who received a low dose of $\mathrm{CD} 34^{+}$cells had significantly fewer episodes of angina per week at 6 months than those in the control group (6.8 vs 10.9 episodes; $\mathrm{p}=0.02$ ) [16]. Weekly angina episodes were also less frequent in the highdose CD $34^{+}$group but the difference was not significant. Improvement in exercise tolerance at 6 months was also significantly greater in the low-dose group than in the placebo group (mean \pm standard deviation (SD): $139 \pm 151$ vs $69 \pm 122 \mathrm{~s} ; \mathrm{p}=0.014)$, and greater, but not significantly in the high-dose group.

Alferminogene tadenovec (Generx ${ }^{\mathrm{TM}}$; Cardium Therapeutics, CA, USA) uses an adenovirus serotype-5 vector to deliver human FGF-4, which induces structural and physiological changes in the heart including the growth of new collateral blood vessels. Alferminogene tadenovec is entering a Phase III trial (ASPIRE) in 100 patients with stable angina who are symptomatic despite optimal medical therapy and who have a reversible perfusion defect of $>9 \%$

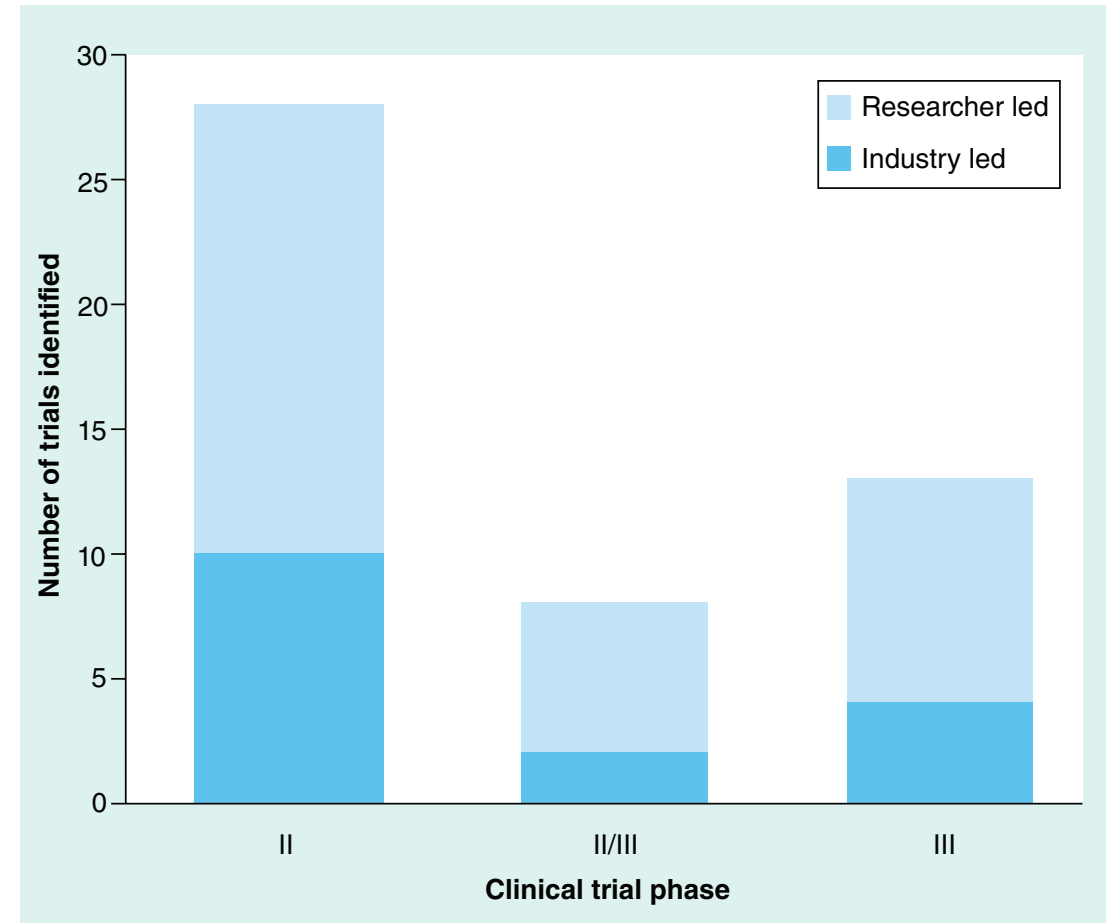

Figure 1. Industry-led versus researcher-led clinical trials by phase. 


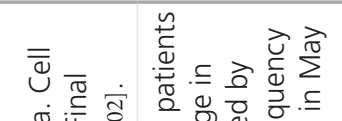

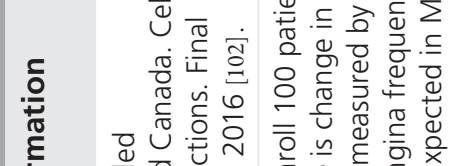

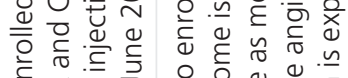

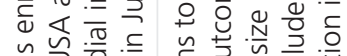

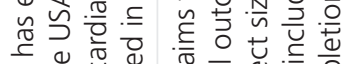

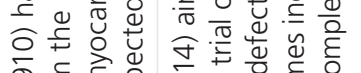

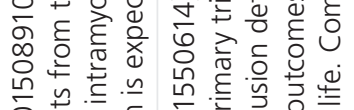

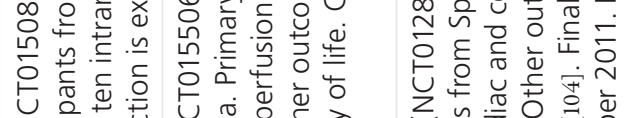

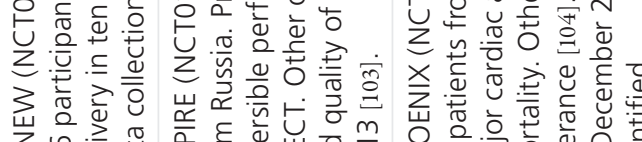

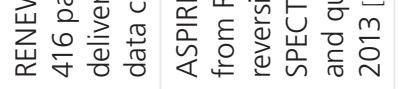

艹

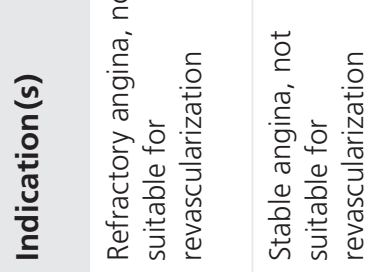

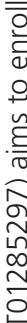

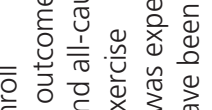

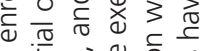

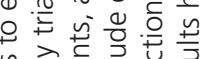

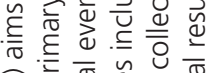

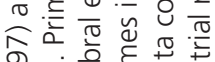

वृ.

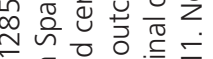

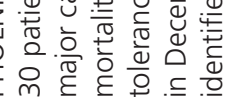

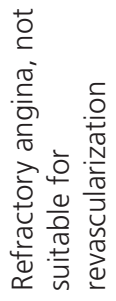

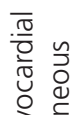

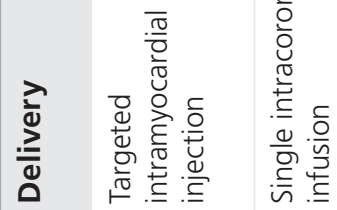

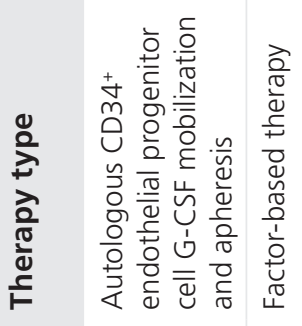

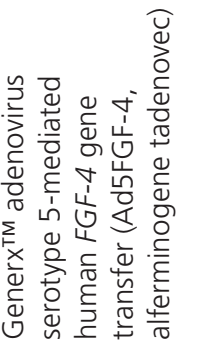

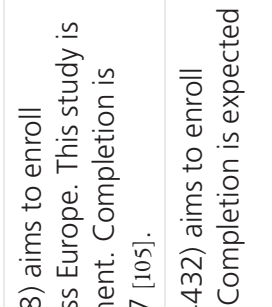

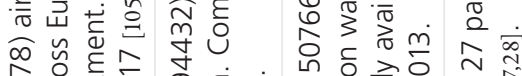

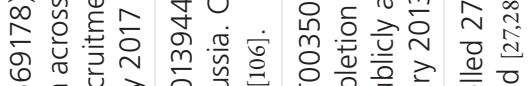

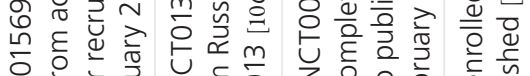

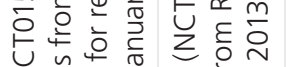

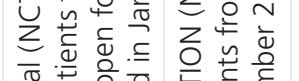

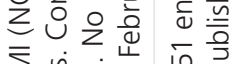

$\sum_{4}$

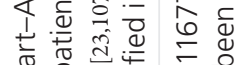

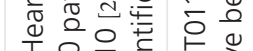

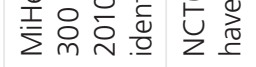

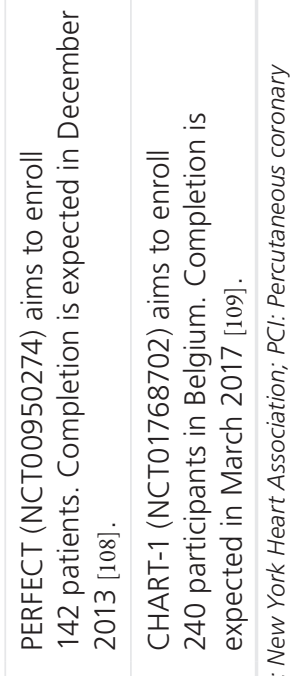

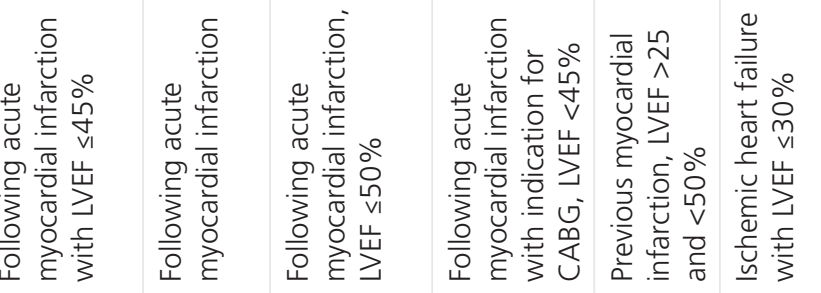

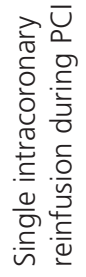

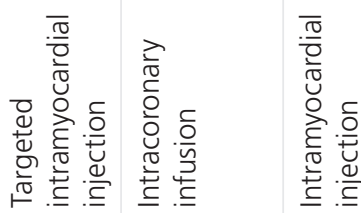

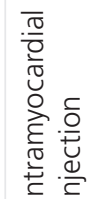

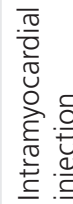

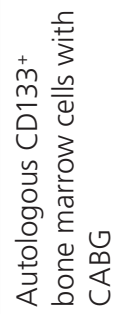

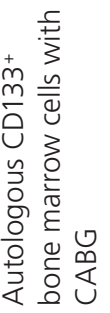

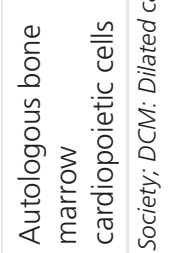

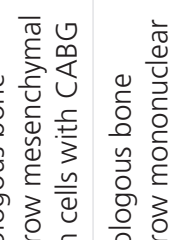

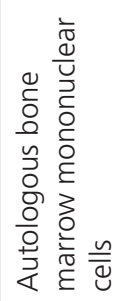

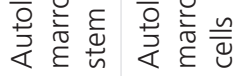

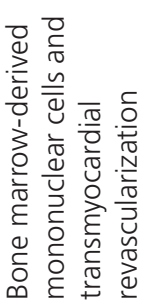

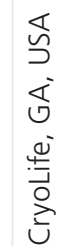

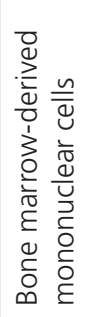

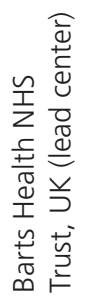

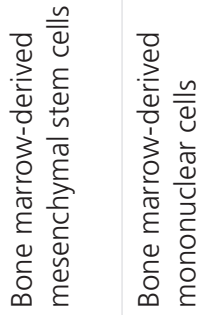

紊

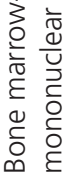

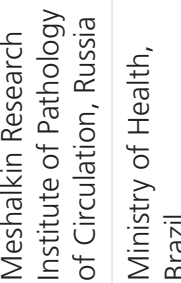

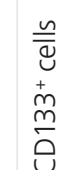

$\sum_{0}^{1} \frac{1}{\sqrt{0}}$

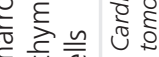

य)

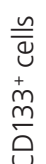

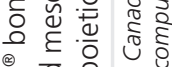

ब

U⿺

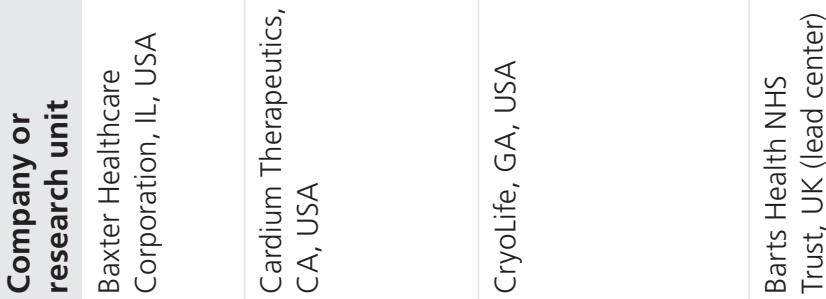

m

४

เก

6

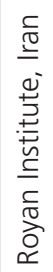

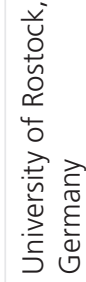

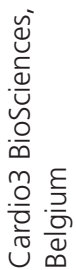




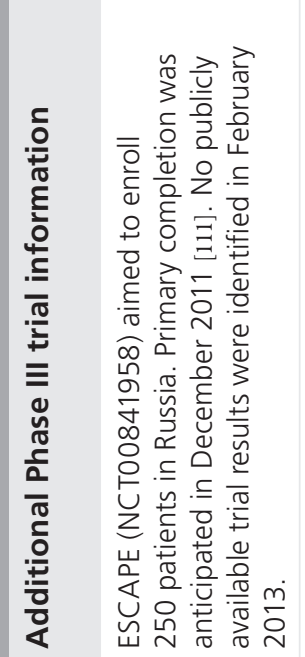

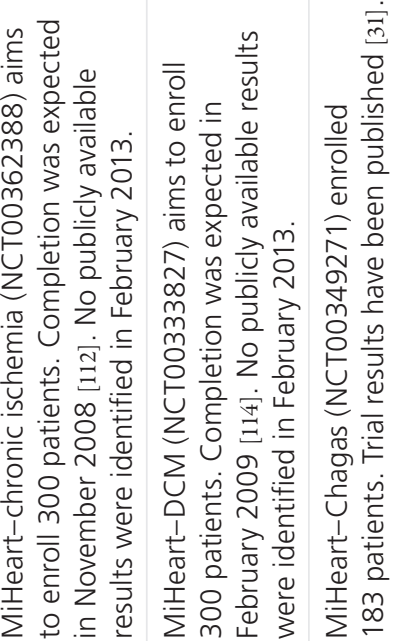

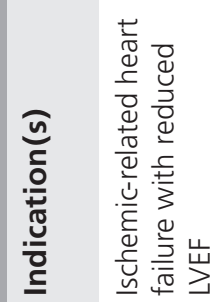

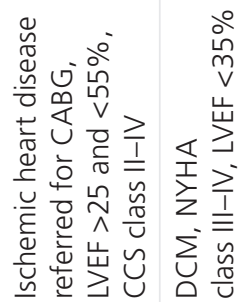<smiles>C1=C[As]C1</smiles>

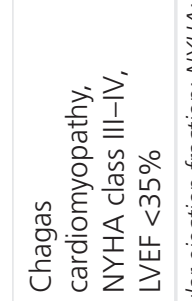

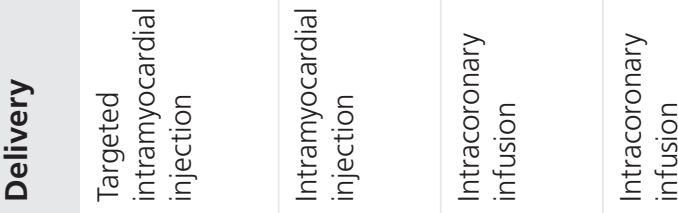

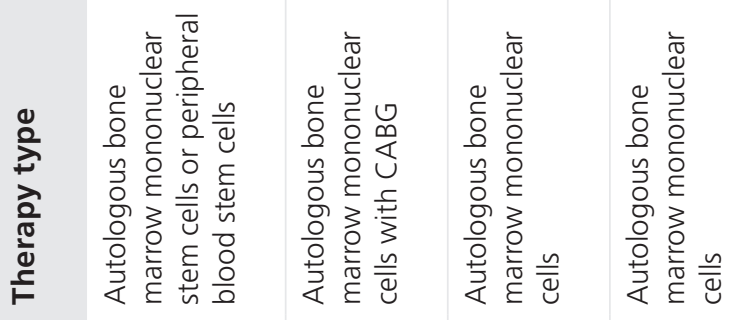

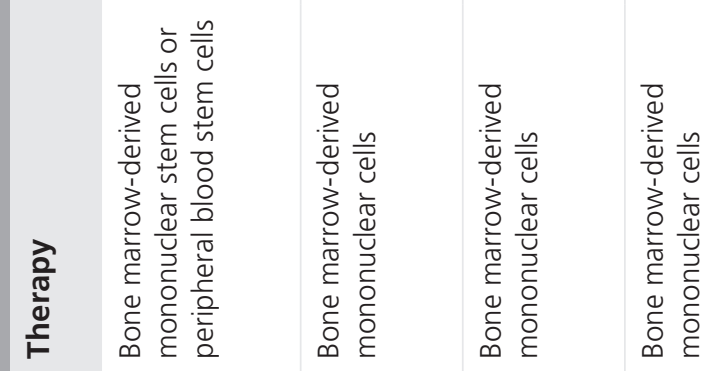

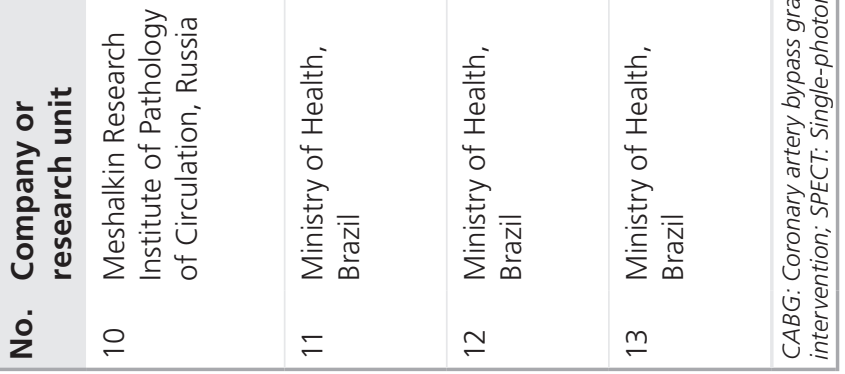


on stress single-photon emission computer tomography [103]. Two Phase IIb/III trials of alferminogene tadenovec (AGENT-3 and AGENT-4) were stopped early when interim analysis of one trial revealed that the study was unlikely to yield statistically significant results [17]. The primary end point was exercise tolerance at 12 weeks. Large and significant placebo effects were found in each trial. A pooled analysis of the Phase IIb/III results suggested that, for a high-dose group there were significant changes in the Canadian Cardiovascular Society angina scale and statistically significant gender differences [17].

CryoLife (GA, USA) are trialing the combination delivery of transmyocardial laser revascularization and autologous bone-marrow derived mononuclear cells. The PHOENIX Phase III trial aims to enroll 30 patients with refractory angina who are not suitable for a percutaneous coronary intervention [104]. In a single-arm, open-label study, the system appeared to provide efficient delivery of concentrated autologous bone marrow-derived mononuclear cells and resulted in reduced angina class (mean $\pm \mathrm{SD}$ : $3.4 \pm 0.5$ vs $1.4 \pm 0.6 ; \mathrm{p}=0.004)$, monthly medication use (mean \pm SD: $348 \pm 118$ vs $201 \pm 92$; $\mathrm{p}=0.001)$ and cardiac-related hospital readmissions (mean \pm SD: $2.9 \pm 2.3$ vs $0.5 \pm 0.8$; $\mathrm{p}<0.001)$ in 19 participants [18]. Although the efficacy of transmyocardial laser revascularization has not been adequately proven, it is hypothesised to enhance homing of cells and stem cell paracrine effects $[19,20]$.

\section{Following myocardial infarction}

A large multicenter Phase III trial of intracoronary infusion of autologous bone marrowderived mononuclear cells following myocardial infarction is in the final planning stages. The BAMI study plans to enroll 3000 patients following myocardial infarction with a reduced LVEF and significant regional wall movement abnormality 3-6 days after reperfusion therapy [105]. The primary outcome will be time to death; secondary outcomes include cardiac mortality, cardiac-related hospitalizations and adverse events. The trial builds on results from smaller Phase II studies including REPAIRAMI, a trial of intracoronary infusion of bonemarrow derived progenitor cells in 204 patients 3-7 days after successful infarct reperfusion therapy. At 4 months the primary end point of absolute improvement in global LVEF was significantly greater with cell therapy than placebo (mean increase \pm SD: $5.5 \pm 7.3 \%$ vs $3.0 \pm 6.5 \%$; $\mathrm{p}=0.01$ ) [21]. At 2 years the cumulative end point of death, myocardial infarction and the need for revascularization was significantly reduced with cell therapy (hazard ratio: 0.58 ; $95 \% \mathrm{CI}$ : 0.36-0.94; $\mathrm{p}=0.025)$ [22].

The ESTIMATION Phase III study will assess the efficacy of intramyocardial injection of autologous bone marrow-derived mesenchymal stem cells in combination with CABG in 50 patients following acute myocardial infarction [106]. The trialists aim to include patients approximately 7-10 days after percutaneous coronary intervention. The primary end point is reduction in left ventricular systolic volume measured by MRI. Secondary end points include mortality, thromboembolic events, hospitalization for heart failure and exercise tolerance. The MiHeart-AMI study in Brazil aimed to recruit 300 patients and administer autologous bone marrow-derived mononuclear stem cells approximately 5-7 days after reperfusion therapy [107]. The primary end point is a change in LVEF at 6 and 12 months. Other end points include major cardiovascular events and quality of life [23].

$\mathrm{CD} 33^{+}$cells are highly proliferative primitive hematopoietic progenitor cells that can be isolated from samples of bone marrow-derived mononuclear cells. The use of $\mathrm{CD} 133^{+}$cells in combination with CABG is supported by encouraging results from early trials that have reported an increase in LVEF [24,25]. CD133+ cells have also been used in trials by intracoronary administration in the week following myocardial infarction [26].

A Phase III randomized trial of autologous bone marrow-derived $\mathrm{CD} 133^{+}$cells in patients following myocardial infarction was completed at the Royan Institute, Iran. In the study 27 participants with a recent myocardial infarction (18-90 days postinfarction) and LVEF <45\% underwent $\mathrm{CABG}$ and an intramyocardial injection of autologous bone marrow-derived $\mathrm{CD}_{133}{ }^{+}$cells or CABG alone. The primary end point was change in LVEF. At 6 months there was no difference in LVEF, however the wall motion score index was significantly reduced for akinetic and dyskinetic segments ( $<<0.006)$ with cellular therapy [27]. At 5 years there was no statistically significant difference in LVEF; however, the improvement in wall motion score index was sustained [28].

The PERFECT Phase III trial is also investigating the intramyocardial injection of CD133+ cells in combination with CABG in a planned 142 patients following myocardial infarction who have an indication for surgical revascularization and a reduced global LVEF (between 25 
and $50 \%)[29,108]$. The primary end point will be LVEF at 6 months. Secondary end points include exercise capacity, angina class, hospitalizations and mortality.

\section{Ischemia-related heart failure}

Cardio3 BioSciences are sponsoring a Phase III European trial (CHART-1) of C-Cure autologous bone marrow-derived mesenchymal cardiopoietic cells in 240 patients with New York Heart Association (NYHA) class III or IV ischemic heart failure who are not in need of revascularization [109]. The mesenchymal stem cells are reprogrammed to become precursors of cardiac muscle cells - cardiopoietic cells - and administered by intramyocardial injection. The primary trial outcome is a composite of mortality; worsening heart failure, as measured by hospitalizations, transplantations, myocardial infarctions and strokes; quality of life; exercise tolerance; and change in left ventricular function at 38 weeks. Secondary safety and efficacy outcomes were measured at 52 and 104 weeks postinjection. Phase II results in 45 patients found a significant increase in LVEF with C-Cure compared with the control group (mean \pm SD: $5.2 \pm 0.6 \%$ vs $1 \pm 0.7 \% ; \mathrm{p}<0.01)[110]$. Exercise tolerance was significantly increased at 6 months post-therapy.

The ESCAPE study assessed the efficacy of intramyocardial autologous bone marrow mononuclear stem cells or peripheral blood stem cells in 250 patients with CVD, low ejection fraction and signs of heart failure. The primary study outcome was survival in the cell group compared with a medical control group [111]. Study completion was anticipated in December 2011. No publicly available results were identified.

The MiHeart-chronic ischemia study in Brazil aimed to recruit 300 patients with severe heart failure referred for CABG and administer autologous bone marrow-derived stem cells [112]. The primary end point is a change in LVEF. Other end points include major cardiovascular events and quality of life. No publicly available results were identified.

\section{Discussion}

Most of the identified cell-based therapies are in development to mitigate the effects of acute and chronic ischemic heart disease and involve the administration of autologous bone marrowderived stems cells. Although the majority of techniques and products are in early-phase trials, 13 are in Phase III. However, of these 13, six are in development in Iran, Russia and Brazil, which may indicate that even if efficacy is demonstrated, licensing and availability in Europe may be delayed. Most of the approaches in Phase III trials have some evidence of possible benefit from earlier-phase trials; except for alferminogene tadenovec (Suplementary Tabies $1 \& 2$ ). The factor-based approach in Phase III trials is unsupported by evidence from earlier trials.

The majority of techniques and products the authors identified use autologous cells. These cells are more acceptable from a patient perspective, have fewer associated adverse effects and are more easily regulated; however, from a commercial perspective they are less desirable owing to personalized manufacturing requirements and scientific complexities. It is therefore not surprising that there are few commercially driven developments in this field; autologous approaches offer little opportunity for commercial gain and allogenic approaches, although offering potential benefits, have unanswered questions about their immune status.

The choice of end points in regenerative medicine trials for CVD is of importance for patients, professionals and those assessing clinical effectiveness and cost-effectiveness. Study primary outcomes in the majority of Phase III trials that were identified include left ventricular function, exercise tolerance and mortality. Although surrogate end points such as left ventricular function may be helpful in assessing whether cellular therapy is efficacious, they are unlikely to be very helpful in discussions with patients and funders about likely future impacts on morbidity and activities of daily living. Although secondary outcomes for current Phase III studies include more patient-centered outcomes such as episodes of angina, major cardiac events such as reinfarction and restenosis requiring revascularization, and episodes of heart failure, many of the studies are unlikely to be powered to identify significant changes in these measures.

This review is the first that has attempted to identify the range of emerging regenerative medicine techniques and products for CVD and investigate their stage of development. The products identified employed a range of techniques, but none had all the elements of the ideal cardiac regenerative therapy suggested by Ptaszek et al. [11].

In planning how to evaluate and implement regenerative medicines, health policymakers will need to understand the intricacies of the products, the range of approaches being researched in the field and the implications of each approach. Although the majority of 
approaches to regenerative medicine the authors identified were stem cell transplants, a few may be regulated as advanced therapy medicinal products by the EMA [30]. Advanced therapy medicinal products, as defined in Directive 2001/83/EC, include gene therapy medicinal products, somatic stem cell medicinal products and tissue-engineered products [113].

This review was conducted using only publicly accessible information and some information provided by univerCellmarket [101]. Neither companies nor investigators were systematically contacted to find information on ongoing development, progress with clinical trials, trial outcomes or to confirm European launch and licensing plans. It is likely that some of the techniques and products identified will no longer be in development, and it is probable that some of the research centers identified as active in this field are no longer involved in this research. It is also possible that the authors have missed some trials, particularly as keywords relating to regenerative medicine seem to be used in an arbitrary manner.

A significant complication arose in how to categorize research activity in relation to trials involving bone marrow-derived mononuclear cells particularly with collaborators in the emerging BAMI trial. Twenty-one centers within Europe are listed as collaborators in the BAMI trial, some of which are included in Supplementary $T_{A B L E} \mathbf{1}$ as being research active in this field.

\section{Future perspective}

As regenerative medicine approaches come to market, the implications for health services and patients may be significant and will need careful planning to ensure access by those who could benefit. For selected patients these approaches may offer ground breaking new treatment options with a potential for the retention or restoration of cardiac function, leading to an increased quality of life and perhaps length of life. However, new patient pathways and services for cell collection, manipulation and reimplantation will be required before the new options can be offered, while the benefits to health services may accrue over time. It is possible that, in a country such as the UK, working in a situation of constrained finances, without a national reimbursement step and with localized commissioning of health services, access to new regenerative medicine techniques may be difficult following licensing or availability. Many of these challenges are outlined within the UK Strategy for Regenerative Medicine and steps to address barriers to implementation and to provide support to companies and stakeholders with an interest in this area are gradually being addressed [4]. It is likely that other countries developing regenerative medicines will look to employ similar strategies.

The broader application of regenerative medicine techniques for patient benefit requires

\section{Executive summary}

\section{Aim}

- The aim of this review was to identify emerging applications of regenerative medicine that have the potential to benefit patients with cardiovascular disease and to investigate their stage of development.

\section{Methods}

- We identified appropriate contacts and organizations in the UK and undertook a detailed online search to identify developments throughout the world. We searched clinical trial databases to gather information on products closer to licensing or launch.

\section{Results}

- We identified 49 Phase II, II/II and III clinical trials of regenerative medicine techniques for a range of cardiovascular diseases. Thirteen of these trials are Phase III, eight are Phase II/III and 28 are Phase II trials.

- The majority of techniques and products are in development for acute myocardial infarction and myocardial ischemia, and involve an intracoronary infusion or intramyocardial injection of autologous bone marrow-derived stem cells. We also identified two allogenic cellular products in Phase II trials and three factor-based therapy products (one in Phase III and two in Phase II clinical trials).

- Most of the approaches in Phase III trials have some evidence of possible benefit from earlier-phase trials.

\section{Conclusion}

- Despite ongoing research activity, translation into clinical practice appears to be some way off. There is also as of yet no agreement on the most beneficial cell type; the timing of cell harvest, preparation and isolation; or the optimal method, timing or site for delivery of cellular or factor-based therapy.

\section{Future perspective}

- Although regenerative medicine may eventually offer a new, additional or complementary treatment option for patients with cardiovascular diseases, its exact clinical role has yet to be defined. As with all new treatments, rigorous clinical trials are required to determine efficacy, safety and effective delivery methods, and research is still required to understand whether current knowledge will translate into clinical applications. 
ongoing scientific discovery and collaboration between basic and translational researchers, commercial interests, the clinical community, health services and governments. Even following positive clinical trials proving efficacy and safety, many issues remain to be addressed, including cell storage and safety, regulation and legislation, ethical implications (although these are reduced with the use of autologous cells), economic evaluation, reimbursement and other funding issues.

Although regenerative medicine may eventually offer a new, additional or complementary treatment option for patients with CVDs, its exact clinical role has yet to be defined. Like all new treatments rigorous clinical trials are required to determine efficacy, safety and effective delivery methods. The development of commercially viable therapies will be complex and research is still required to understand how current knowledge will translate into clinical applications. Consideration is also needed as to how these techniques will fit in the innovation pathway, how evaluating bodies will appraise regenerative interventions, and how they will be funded, delivered and commissioned. The identification of techniques and products on the horizon such as that undertaken in this review

\section{References}

Papers of special note have been highlighted as:

" of interest

" " of considerable interest

1 Murata M, Tohyama, Fukada K. Impacts of recent advances in cardiovascular regenerative medicine on clinical therapies and drug discovery. Pharmacol. Ther. 126, 109-118 (2010).

2 Alexander JM, Bruneau BG. Lessons for cardiac regeneration and repair through development. Trends Mol. Med. 16, 426-434 (2010).

3 Department for Business Innovation and Life Skills. Taking Stock of Regenerative Medicine in the United Kingdom. Department for Business Innovation and Life Skills, Sheffield, UK (2011).

- Describes the UK's leading position in the regenerative medicine field and the current regulatory system, and the challenges that the $\mathrm{UK}$ is likely to face in relation to regenerative medicines.

4 Medical Research Council. A Strategy for UK Regenerative Medicine. Medical Research Council, London, UK (2012).

- Describes the current position of the UK in the regenerative medicine field and identifies eight strategic objectives that will need to be

will help policymakers plan for the evaluation and adoption of successful therapies by health services.

\section{Disclaimer}

The NIHR did not participate in study design, data collection, data analysis, data interpretation or writing of the report. The corresponding authors had full access to all the data in the study and had final responsibility for the decision to submit for publication.

\section{Financial \& competing interests disclosure}

The National Institute for Health Research Horizon Scanning Centre is a research program funded by the National Institute for Health Research (NIHR). The authors have no other relevant affiliations or financial involvement with any organization or entity with a financial interest in or financial conflict with the subject matter or materials discussed in the manuscript apart from those disclosed.

No writing assistance was utilized in the production of this manuscript.

\section{Open Access}

This work is licensed under the Creative Commons Attribution-NonCommercial 3.0 Unported License. To view a copy of this license, visit http://creativecommons.org/ licenses/by-nc-nd/3.0/

addressed to make the most of its current position and to overcome the obstacles that those developing regenerative medicine technologies are likely to face.

5 Moran N, Sheridan C, Winter P. Regenerate the Future. Science Business Publishing Ltd., Middlesex, UK (2012).

6 Future Medicine. World stem cell report. Global update: USA. Regen. Med. 7(Suppl. 6), 126-129 (2012).

7 Future Medicine. World stem cell report. Global update: Canada. Regen. Med. 7(Suppl. 6), 132-135 (2012).

8 Future Medicine. World stem cell report. Global update: UK. Regen. Med. 7(Suppl. 6), 136-138 (2012).

9 Future Medicine. World stem cell report. Global update: Sweden. Regen. Med. 7(Suppl. 6), 140-142 (2012).

10 Future Medicine. World stem cell report. Global update: Brazil. Regen. Med. 7(Suppl. 6), 144-147 (2012).

11 Ptaszek LM, Mansour M, Ruskin JN, Chien KR. Towards regenerative therapy for cardiac disease. Lancet 379, 933-942 (2012).

12 Vassalli G, Moccetti T. Cardiac repair with allogenic mesenchymal stem cells after myocardial infarction. Swiss Med. Wkly 141, w13209 (2011).
13 Kinkaid HY, Huang XP, Li RK, Weisel RD. What's new in cardiac cell therapy? Allogeneic bone marrow stromal cells as 'universal donor cells'. J. Card. Surg. 25(3), 359-366 (2010).

14 Hare JM, Fishman JE, Gerstenblith G et al. Comparison of allogeneic vs autologous bonemarrow-derived mesenchymal stem cells delivered by transendocardial injection in patients with ischemic cardiomyopathy. The POSEIDON randomized trial. JAMA 308(22), 2369-2379 (2012).

15 Clifford DM, Fisher SA, Brunskill SJ et al. Stem cell treatment for acute myocardial infarction. Cochrane Database Syst. Rev. 2, CD006536 (2012).

- Update of a previous article (published in 2008) that reviewed randomized controlled trials comparing autologous stem/progenitor cells with no autologous stem/progenitor cells in patients diagnosed with acute myocardial infarction.

16 Losordo DW, Henry TD, Davidson C et al. Intramyocardial, autologous $\mathrm{CD} 34^{+}$cell therapy for refractory angina. Am. Heart J. 109, 428-436 (2011).

17 Henry TD, Grines CL, Watkins MW et al. Effects of Ad5FGF-4 in patients with angina. J. Am. Coll. Cardiol. 50, 1038-1046 (2007).

18 Reyes G, Allen KB, Alvarez P et al. Mid term results after bone marrow laser 
revascularization for treating refractory angina. BMC Cardiovasc. Disord. 10, 1-6 (2010).

19 NICE. Transmyocardial Laser Revascularization for Refractory Angina Pectoris. NICE, London, UK (2009).

20 Briones E, Lacalle JR, Marin I. Transmyocardial laser revascularisation versus medical therapy for refractory angina. Cochrane Database Syst. Rev. 21(1), CD003712 (2009).

21 Schächinger V, Erbs S, Elsässer A et al. Intracoronary bone marrow-derived progenitor cells in acute myocardial infarction. N. Engl. J. Med. 355, 1210-1221 (2006).

22 Assmus B, Rolf A, Erbs S et al. Clinical outcome 2 years after intracoronary administration of bone marrow-derived progenitor cells in acute myocardial infarction. Circ. Heart Fail. 3, 89-96 (2010).

23 Dohmann HFR, Silva SA, Sousa ALS et al. Multicenter double blind trial of autologous bone marrow mononuclear cell transplantation through intracoronary injection post acute myocardium infarction - MiHeart/AMI study. Trials 9, 41 (2008).

24 Stamm C, Kleine HD, Choi YH et al. Intramyocardial delivery of $\mathrm{CD} 133^{+}$bone marrow cells and coronary artery bypass grafting for chronic ischemic heart disease: safety and efficacy studies. J. Thorac. Cardiovasc. Surg. 133, 717-725 (2007).

25 Klein HM, Assmann A, Lichtenberg A, Heke M. Intraoperative $\mathrm{CD} 133^{+}$cell transplantation during coronary artery bypass grafting in ischemic cardiomyopathy. Multimed. Man. Cardiothorac. Surg. doi:10.1510/mmcts.003947 (2009) (Epub ahead of print).

26 Mansour S, Roy DC, Bouchard V et al. One-year safety analysis of the COMPAREAMI trial: comparison of intracoronary injection of $\mathrm{CD} 133^{+}$bone marrow stem cells to placebo in patients after acute myocardial infarction and left ventricular dysfunction. Bone Marrow Res. 2011, 385124 (2011).

27 Ahmadi H, Baharvand H, Ashtiani SK et al. Safety analysis and improved cardiac function following local autologous transplantation of $\mathrm{CD} 133^{+}$enriched bone marrow cells after myocardial infarction. Curr. Neurovasc. Res. 4(3), 153-160 (2007).

28 Ahmadi H, Moshkani Farahani M, Kouhkan A et al. Five-Year follow-up of the local autologous transplantation of CD133+ enriched bone marrow cells in patients with myocardial infarction. Arch. Iran. Med. 15(1), 32-35 (2012).

29 Donndorf P, Kaminski A, Tiedemann G, Kundt G, Steinhoff G. Validating intramyocardial bone marrow stem cell therapy in combination with coronary artery bypass grafting, the PERFECT Phase III randomized multicenter trial: study protocol for a randomized controlled trial. Trials 13 , 99 (2012).

30 EMA. Advanced therapy medicinal products and the Committee for Advanced Therapies. EMA, London, UK (2013).

- Establishes the European regulatory pathway for regenerative medicine techniques and products.

31 dos Santos RR, Rassi S, Feitosa G et al. Cell therapy in chagas cardiomyopathy (Chagas Arm of the Miheart Study): a multicenter randomized trial. Circulation. 125(20), 2454-2461 (2012).

\section{Websites}

101 univerCELLmarket.

www.univercellmarket.com

(Accessed 19 April 2012)

102 ClinicalTrials.gov. Efficacy and safety of targeted intramyocardial delivery of auto CD $34^{+}$stem cells for improving exercise capacity in subjects with refractory angina (RENEW).

www.clinicaltrials.gov/ct/show/ NCT01508910

(Accessed 14 February 2013)

103 ClinicalTrials.gov. Efficacy and safety of Ad5FGF-4 for myocardial ischemia in patients with stable angina due to coronary artery disease (ASPIRE).

www.clinicaltrials.gov/ct2/show/NCT015506 14 ? term $=$ ASPIRE $\&$ fund $=2 \&$ rank $=5$ (Accessed 14 February 2013)

104 ClinicalTrials.gov. Safety study of transmyocardial revascularization (TMR) with bone marrow aspirate (BMAC) for angina reduction (PHOENIX). www.clinicaltrials.gov/ct2/show/NCT012852 97 ?term $=$ nct $01285297 \&$ rank $=1$ (Accessed 14 February 2013)

105 ClinicalTrials.gov. BAMI. The effect of intracoronary reinfusion of bone marrowderived mononuclear cells (BM-MNC) on all cause mortality in acute myocardial infarction. www.clinicaltrials.gov/ct2/show/NCT015691 78 ? term $=$ NCT01569178 \& rank $=1$ (Accessed 14 February 2013)
106 ClinicalTrials.gov. ESTIMATION study for endocardial mesenchymal stem cells implantation in patients after acute myocardial infarction. www.clinicaltrials.gov/ct2/show/NCT01394 432? term $=$ Estimation \& rank=2 (Accessed 14 February 2013)

107 ClinicalTrials.gov. Cell therapy in myocardial infarction (EMRTCC). www.clinicaltrials.gov/ct2/show/NCT00350 766? term $=$ NCT00350766\& rank $=1$ (Accessed 14 February 2013)

108 ClinicalTrials.gov. Intramyocardial transplantation of bone marrow stem cells in addition to coronary artery bypass graft (CABG) surgery (PERFECT). www.clinicaltrials.gov/ct2/show/NCT00950 274? term $=$ NCT00950274\&rank=1 (Accessed 14 February 2013)

109 ClinicalTrials.gov. Safety and efficacy of autologous cardiopoietic cells for treatment of ischemic heart failure (CHART1). www.clinicaltrials.gov/ct2/show/NCT017687 02? term $=$ NCT01768702\&rank $=1$ (Accessed 14 February 2013)

110 Cardio3 BioSciences. www.c3bs.com/en/products-pipeline/clinical. html

(Accessed 14 February 2013)

111 ClinicalTrials.gov. Endocardial stem cells approach efficacy (ESCAPE). www.clinicaltrials.gov/ct2/show/ NCT00841958? term $=$ ESCAPE \& rank $=9$ (Accessed 14 February 2013)

112 ClinicalTrials.gov. Cell therapy in chronic ischemic heart disease. www.clinicaltrials.gov/ct2/show/NCT00362 388? term $=$ NCT00362388\& $\&$ rank $=1$ (Accessed 14 February 2013)

113 Regulation (EC) no 1394/2007 of the European parliament and of the council of 13 November 2007 on advanced therapy medicinal products and amending Directive 2001/83/EC and Regulation (EC) No $726 / 2004$.

http://eur-lex.europa.eu/LexUriServ/ LexUriServ.do?uri=OJ:L:2007:324:0121:013 7:en:PDF

- Sets standards of quality and safety for the donation, procurement, testing, processing, preservation, storage and distribution of human tissues and cells.

114 ClinicalTrials.gov. Cell therapy in dilated cardiomyopathy. www.clinicaltrials.gov/ct2/show/NCT00333 827 ? term $=$ NCT00333827\& rank $=1$ (Accessed 14 February 2013) 